\title{
Daily Plasmodium yoelii infective mosquito bites do not generate protection or suppress previous immunity against the liver stage
}

Tzvi Pollock ${ }^{1 \dagger}$, Ricardo Leitao ${ }^{1 \dagger}$, Cristina Galan-Rodriguez ${ }^{1}$, Kurt A Wong ${ }^{1,2}$ and Ana Rodriguez ${ }^{1 *}$

\begin{abstract}
Background: Human populations that are naturally subjected to Plasmodium infection do not acquire complete protection against the liver stage of this parasite despite prolonged and frequent exposure. However, sterile immunity against Plasmodium liver stage can be achieved after repeated exposure to radiation attenuated sporozoites. The reasons for this different response remain largely unknown, but a suppressive effect of blood stage Plasmodium infection has been proposed as a cause for the lack of liver stage protection.
\end{abstract}

Methods: Using Plasmodium yoelii 17XNL, the response generated in mice subjected to daily infective bites from normal or irradiated mosquitoes was compared. The effect of daily-infected mosquito bites on mice that were previously immunized against $P$. yoelii liver stage was also studied.

Results: It was observed that while the bites of normal infected mosquitoes do not generate strong antibody responses and protection, the bites of irradiated mosquitoes result in high levels of anti-sporozoite antibodies and protection against liver stage Plasmodium infection. Exposure to daily infected mosquito bites did not eliminate the protection acquired previously with a experimental liver stage vaccine.

Conclusions: Liver stage immunity generated by irradiated versus normal $P$. yoelii infected mosquitoes is essentially different, probably because of the blood stage infection that follows normal mosquito bites, but not irradiated. While infective mosquito bites do not induce a protective liver stage response, they also do not interfere with previously acquired liver stage protective responses, even if they induce a complete blood stage infection. Considering that the recently generated anti-malaria vaccines induce only partial protection against infection, it is encouraging that, at least in mouse models, immunity is not negatively affected by subsequent exposure and infection with the parasite.

\section{Background}

Malaria is a widespread mosquito-borne infectious disease that kills almost one million persons per year, most of which are children. Growing scientific knowledge and the recent malaria research trend provide unparalleled opportunities to develop a malaria vaccine. Although the subunit vaccines are the most advanced in research pipelines and field clinical trials [1], an effective and focused effort prevails in order to develop an attenuated sporozoite-based vaccine $[2,3]$, once the protective

\footnotetext{
* Correspondence: Ana.Rodriguez@nyumc.org

† Contributed equally

'Department of Microbiology, Division of Medical Parasitology, New York

University School of Medicine, New York, NY 10010, USA

Full list of author information is available at the end of the article
}

immunity produced by attenuated sporozoites is stronger and more unswerving than that induced by subunit vaccination [4]. Immunization with irradiated Plasmodium sporozoites grants protection against inoculation with non-attenuated sporozoites in mice models and humans [5,6]. However, individuals in malaria endemic regions do not develop fully protective immune responses against Plasmodium liver stage infections even if exposed to more than two infective mosquito bites daily $[7,8]$, as is the case in high transmission areas [9]. Using a mouse model, a previous study showed that the mode of irradiated sporozoite delivery: daily mosquito bites (as in endemic areas) versus few large doses of sporozoites (as in immunization protocols) does not affect the generation of protective liver stage immunity

\section{Biomed Central}

(c) 2011 Pollock et al; licensee BioMed Central Ltd. This is an Open Access article distributed under the terms of the Creative Commons Attribution License (http://creativecommons.org/licenses/by/2.0), which permits unrestricted use, distribution, and reproduction in any medium, provided the original work is properly cited. 
[10]. In this work, it was assessed whether the daily bites of non-irradiated infected mosquitoes would also induce a protective liver stage immune response, similar to that induced by irradiated infected mosquito bites. This situation would be analogous to the circumstances of humans in high-transmission malaria endemic areas, where people are subjected to daily bites of infected mosquitoes [9].

In this work, it was observed that daily bites of nonirradiated infected mosquitoes do not induce liver stage protection, while the daily bites of irradiated mosquitoes do. This result again confirms the concordance of observations in mice and in humans regarding liver stage immunity and focuses the questions about generation of liver stage protection on the differences between infection generated by irradiated versus normal sporozoites.

Since the use of partially protective malaria liver stage vaccines in the field may soon be a reality [11], how would infected mosquito bites affect the immune response and protection induced by the vaccine becomes a highly relevant question. An enhancing effect caused by additional boosting liver stage antigens could be expected, however, an inhibition of the vaccineinduced protection has also been proposed [12]. Therefore, a mouse model was used to test whether the bites of infected mosquitoes could affect previously acquired liver stage immunity. Interestingly, it was found that after immunization of the mice, daily bites of infected mosquitoes do not significantly affect the liver stage protective response.

\section{Methods}

Parasites, mosquitoes and mouse immunizations

Anopheles stephensii mosquitoes were maintained as described [13] and infected with P. yoelii 17XNL. This parasite line has been grown for over ten years without cloning and is the same used in previous work [10]. Irradiated mosquitoes were generated by exposure to 12 krad (120 Gy) of $\gamma$-irradiation (MDS Nordion Gammacell 1000 Elite). Female Swiss-Webster mice (NIH, Bethesda MD) were used for all experiments. For the partial immunization, each mouse was injected intravenously with 75,000 P. yoelii $17 \mathrm{XNL}$ irradiated (12 krad) sporozoites dissected from the salivary glands of infected mosquitoes, followed two weeks later with a dose of 50,000 . For daily mosquito bites, each mouse had two mosquitoes feed on the tail for $3 \mathrm{~min}$. This was repeated daily for 6 weeks. Parasitaemia was followed regularly using thin blood smears from a drop of tail blood.

\section{Immunofluorescence titration of serum antibodies}

For titration of $P$. yoelii-specific antibody levels, $10^{4}$ salivary-gland sporozoites in each well were air-dried on glass multiwell IFA slides. Mouse serum was titrated and primary antibody bound to sporozoites was detected using FITC-labelled antimouse IgG (Sigma). A monoclonal P. yoelii CS-specific antibody (2F6) was used as a positive control (kindly provided by Dr. Photini Sinnis, NYU).

\section{Challenge and quantitative real-time PCR}

Challenge of mice was performed in groups of five mice by i.v. injection of $10^{4}$ (non-irradiated) P. yoelii 17XNL GFP sporozoites [14], freshly dissected from mosquito salivary glands. Forty hours later, livers were harvested, total RNA was isolated, and malaria infection was quantified using reverse transcription followed by real-time PCR with primers that recognize GFP-specific sequences: 5'-GTC AGT GGA GAG GGT GAA GG-3' and 5'-ACT TCA GCA CGT GTC TTG TAG TTC-3'. Reaction was run for 40 cycles. Threshold cycle is the fractional cycle at which the fluorescence signal passes the threshold, which was set up at $50 \%$ of the maximal fluorescence signal.

\section{Statistical analysis}

Data were analyzed using Prism (v. 4.0c, GraphPad). ANOVA test was performed as mentioned. Statistics were considered significant if $P<0.05$.

\section{Results and discussion}

To compare the responses of mice to daily normal or irradiated mosquito bites, a protocol previously shown to induce protection in mice when mosquitoes infected with the non-lethal parasite $P$. yoelii $17 \mathrm{XNL}$ are irradiated was followed [10]. The first group received an immunization regimen of daily bites from two irradiated mosquitoes to mimic an entomological inoculation rate found in regions of very high transmission $[9,15]$. The second group received identical treatment, but mice were bitten by non-irradiated infected mosquitoes. For both groups, each mouse had two mosquitoes feed on her tail for three minutes. This was repeated daily for six weeks (Figure 1).

To study whether the immune response induced by a vaccine can be affected by subsequent bites of infected mosquitoes, we immunized mice with two doses of radiation-attenuated sporozoites. This immunization protocol that induces only partial liver stage protection was purposely chosen to reflect the situation with current human liver stage vaccine prototypes [11]. After immunization, mice were subjected or not to daily bites of infected mosquitoes, as described above (Figure 1).

One week after the last mosquito bite a small volume of serum was obtained from each mouse to determine anti-sporozoite antibody levels and seven weeks later mice were challenged with non-irradiated P. yoelii sporozoites to determine liver stage protection. 


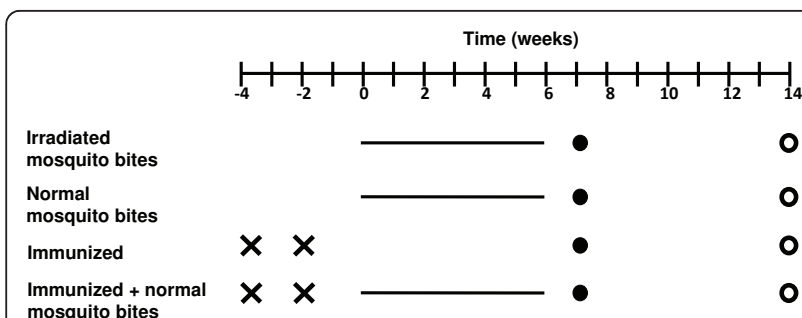

Figure 1 Experiment schedule. Four groups of five mice received different immunizations and were subjected to the bites of irradiated or normal infected mosquito bites as indicated. Two groups were immunized by intravenous injection of two doses of irradiated sporozoites two weeks apart (represented by crosses in the figure). After another two weeks, three of the four groups were subjected to two daily bites of infected mosquitoes, irradiated (dashed line) or not (continuous line) for six weeks. One week later (black circles), a small volume of serum was extracted from each mouse to determine anti-sporozoite antibody levels (results shown in Figure 3). Eight weeks later mice were challenged (white circles) and liver stage infection detected with real-time PCR (results shown in Table 1).

Parasitaemia was measured in each mouse weekly, confirming that irradiation of sporozoites and mosquitoes was effective; no blood stage infection was found in the group subjected to the bites of irradiated mosquitoes or the group immunized with irradiated sporozoites that did not receive mosquito bites. The data also show that mosquito bites induce a delayed development and lower levels of parasitaemia in mice that were previously immunized with irradiated sporozoites compared to non-immunized mice, probably reflecting the lower liver stage infection levels in the partially immunized mice (Figure 2).

When the levels of anti-sporozoite antibodies were determined, we observed that mice subjected to the bites of irradiated, infected mosquitoes develop significantly higher levels of antibodies compared to mice subjected to the bites of normal infected mosquitoes. It is important to note that both groups of mice had received equal numbers of mosquito bites and it is, therefore, expected that each group had received equal numbers of sporozoites.

Antibody levels in the groups of mice that were immunized with two doses of the irradiated sporozoite vaccine followed or not by the bites of normal infected mosquitoes showed no significant differences in their anti-sporozoite antibody levels, suggesting that after immunization, the antibody levels are not affected by the bites of infected mosquitoes (Figure 3), even if the mice developed a full blood stage infection (Figure 2).

To study the level of immunization acquired by the different groups of mice, each mouse was challenged

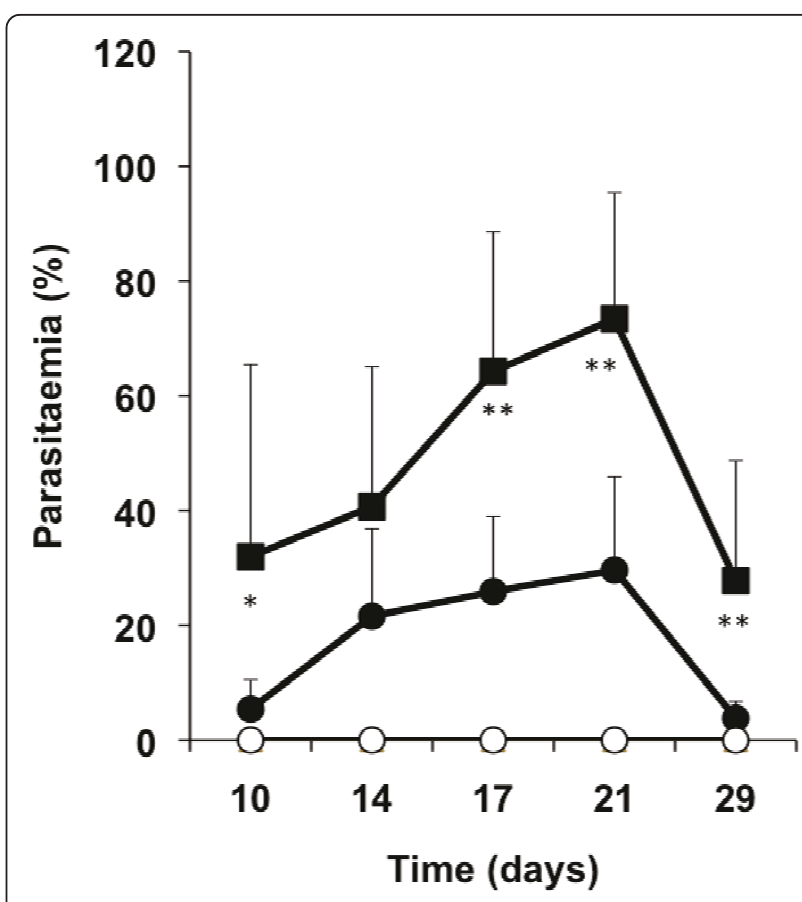

Figure 2 Determination of parasitaemia. Parasitaemia was determined in peripheral blood in all groups of mice every other day, starting ten days after the beginning of daily mosquito bites. Average parasitaemias are shown for each group. Parasitaemia in the group subjected to normal mosquito bites (black squares) and immunized followed by normal mosquito bites (black circles) are shown. In the groups subjected to irradiated mosquito bites (white circles) and immunized control (white squares) no infected erythrocytes were detected. Error bars represent standard deviation within groups of mice ${ }^{*}, P<0.05 ;{ }^{*}, P<0.01$, when comparing mice subjected to normal mosquito bites and immunized followed by normal mosquito bites mice using ANOVA).

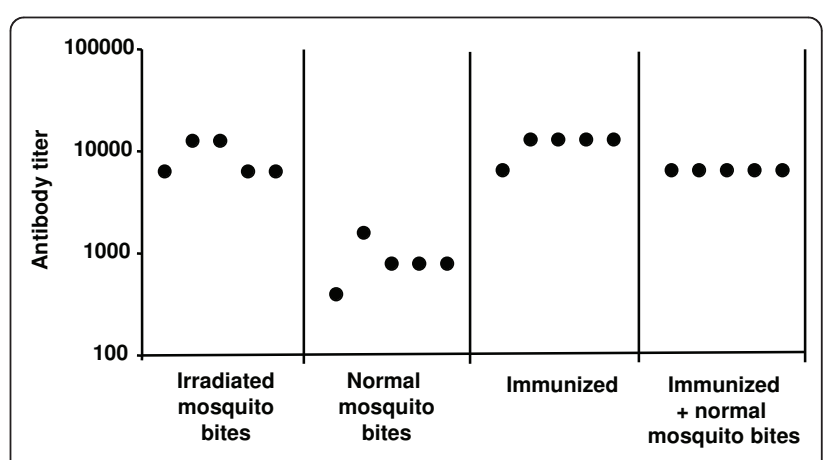

Figure 3 Anti-sporozoite antibody titres. Serum from each mouse was serially diluted and used to stain $P$. yoelii sporozoites. The titers represent the inverse of the highest dilution at which sporozoites could still be detected. Each circle represents an individual mouse. Significant differences between the group of normal mosquito bite and the other groups were found $(P<0.05)$ when comparing with ANOVA). 
with non-irradiated $P$. yoelii-GFP sporozoites. It is important to use a $P$. yoelii strain that allows us to differentiate the challenge parasites from parasites (in liver or blood stage forms) that were inoculated before by either the immunization protocols or the mosquito bites. This is possible analysing the livers of challenged mice using real-time PCR to amplify the GFP sequence present only the challenge sporozoites. This technique is not as sensitive as the conventional RT-PCR used for detection of liver stage infection, which amplifies sequences within the $18 \mathrm{~S}$ rRNA that are very abundant in the parasite [16].

Mice were challenged 14 weeks after being subjected to the first infected mosquito bite, when blood stage parasitaemia was undetectable. It was found that, while the liver stages of the GFP challenge sporozoites were not detectable in mice subjected to irradiated infected mosquito bites, mice that had been bitten by normal infected mosquitoes developed a detectable liver stage infection (Table 1). These results indicate a higher level of liver stage protection in mice subjected to irradiated infected mosquito bites. In this study the challenge was performed by i.v. injection of sporozoites, which is different from the natural environment, where mosquito bite inoculation would deposit sporozoites in the skin of the host. Despite these differences, the results observed in mice reflect the situation of humans, which are also protected by the bites of irradiated infected mosquitoes [5], but are not protected by natural exposure to infected mosquito bites [7]. It should be noted that this study was performed with two $P$. yoelii-infected mosquito bites per day, which is considered a high transmission rate and, therefore, it is not possible to extrapolate the results to lower frequencies of transmission, to a situation with concurrent bites of infected and uninfected mosquitoes or to infection with different strains of Plasmodium, which would reflect more accurately the conditions in malaria-endemic areas. In particular, the degree of variability of Plasmodium strains that is found in the field may interfere with the protection induced by irradiated infected mosquitoes.

\section{Table 1 Protection against challenge}

\begin{tabular}{lcc}
\hline $\begin{array}{l}\text { Mice experimental } \\
\text { conditions }\end{array}$ & $\begin{array}{c}\text { Threshold cycle (Ct) in } \\
\text { real time-PCR }\end{array}$ & $\begin{array}{c}\text { Number of mice } \\
\text { (protected/total) }\end{array}$ \\
\hline $\begin{array}{l}\text { Irradiated mosquito } \\
\text { bites }\end{array}$ & $>40$ & $(3 / 3)$ \\
$\begin{array}{l}\text { Normal mosquito } \\
\text { bites }\end{array}$ & $17 \pm 0.85$ & $(0 / 3)$ \\
Immunized & $>40$ & $(3 / 3)$ \\
Immunized + normal & $>40$ & $(3 / 3)$ \\
mosquito bites & & \\
\hline
\end{tabular}

Groups of mice were challenged with $10^{4} \mathrm{P}$. yoelii-GFP sporozoites injected intravenously. Real-time PCR was run for 40 cycles, therefore $\mathrm{Ct}>40$ indicates no detection of parasite in the liver.
In groups of mice that were immunized with irradiated sporozoites, subsequent infected mosquito bites, which caused a patent blood stage infection (Figure 2), did not induce a loss of the protection acquired with the experimental vaccination (Table 1 ). These results indicate that liver stage protection induced by irradiated sporozoites is not significantly affected by subsequent infection in mice. It is important to note that the groups of mice that were partially immunized with irradiated sporozoites probably developed a low-level liver stage infection. However, this level of infection is not detected by the GFP RT-PCR used in this assay.

Taken together, these data indicate that the generation of liver stage immunity against malaria is greatly dependent on whether the mosquitoes that transmit infection are irradiated or not. For both mice and humans, it has been extensively demonstrated that irradiated sporozoites induce a protective immune response $[5,6]$, however, what remains unclear is why normal liver stage infection does not induce comparable protection. The possibility that low doses of sporozoites delivered in the skin by mosquito bites could induce tolerance to this stage of the parasite has been discarded in the mouse model [10]. It has been also proposed that the subsequent blood stage infection that follows the liver infection interferes with the generation of protection to the liver stage [12], a mechanism that could underlie the lack of protection observed in mice beaten by infective mosquitoes. It is possible that the high inflammatory immune response that blood stage infection generates could affect the establishment of a competent liver stage memory response that would be maturing at the same time as the blood stage is at its peak. It is also possible that the aborted liver infection that takes place with irradiated sporozoites generates a more protective immune response compared to liver infection by regular sporozoites. Previous experiments in mice suggest that this is not the case, because the liver stage response was found to be similar in mice infected with irradiated sporozoites compared to mice infected with normal sporozoites and treated to inhibit blood stage infection [12]. However, in this work [12] the response was only measured for CS protein and it is possible that the immune response to other proteins involved in Plasmodium liver stage protection [17] could be different when induced by irradiated versus regular sporozoites.

The analysis of mice that were first partially immunized with irradiated sporozoites and then subjected or not to infected mosquito bites indicates that liver stage protection was not lost after infective mosquito bites that result in blood stage infection. Since the mice were only partially protected, a full blood stage infection took place after mice were bitten by infective mosquitoes. This allowed us to analyze the effect of blood stage 
infection on the maintenance of protection induced by the vaccination. Contrarily to the immune suppression described when blood stage infection takes place right after the liver stage [12], the protection induced by the vaccination in our mice was not lost after blood stage infection that occurred four weeks after the first immunization (two weeks after the last immunization). These results suggest that after establishment of the protective liver stage immune response, this response is not significantly affected by subsequent blood stage infection.

\section{Conclusions}

The results presented show that the liver stage immunity generated in response to irradiated versus normal $P$. yoelii infected mosquitoes is essentially different: while irradiated mosquitoes induce a protective immune response with high levels of anti-sporozoite antibodies, normal infected mosquitoes do not generate protection and only low levels of specific antibodies. This situation reflects the response of humans observed both in vaccination trials, where irradiated mosquito bites induce protection, and in the field, where years of constant re-infections do not result in sterile protection against the liver stage. The direct comparison of the human immune response to normal versus irradiated mosquito bites is not possible because the conditions of exposure to infective mosquito bites in the field are very different from experimental exposure to irradiated mosquito bites. In directly comparing the response generated by irradiated or non-irradiated infected mosquito bites, this work indicates that the mosquito irradiation is the only essential variable needed to generate protective liver stage response.

While infective mosquito bites do not induce a protective liver stage response, they also do not interfere with previously acquired liver stage protective responses, even if they induce a complete blood stage infection. Considering that the recently generated anti-malaria vaccines induce only partial protection against infection, it is encouraging that, at least in mouse models, immunity is not negatively affected by subsequent exposure and infection with the parasite.

\section{Acknowledgements and funding}

Authors would like to acknowledge Sandra Gonzalez for her help in providing the infected mosquitoes. R.L. is the recipient of fellowship from Fundacao para a Ciencia e Tecnologia, Portugal. A.R. was supported by NIH grant R01 Al 053698

\section{Author details}

${ }^{1}$ Department of Microbiology, Division of Medical Parasitology, New York University School of Medicine, New York, NY 10010, USA. ${ }^{2}$ Current Address: The Scripps Research Institute, La Jolla, CA 92037, USA.

\section{Authors' contributions}

TP performed mice mosquito daily bite infections and measured parasitaemias. RL carried out mice immunizations, real-time PCR experiments and contributed to the writing of the manuscript. CGR carried out antibody quantifications. KAW conceived the study, participated in its design and trained TP. AR conceived the study, participated in its design and coordination and wrote the manuscript. All authors read and approved the final manuscript.

\section{Competing interests}

The authors declare that they have no competing interests.

Received: 15 December 2010 Accepted: 18 April 2011

Published: 18 April 2011

\section{References}

1. Aide P, Aponte JJ, Renom M, Nhampossa T, Sacarlal J, Mandomando I, Bassat Q, Manaca MN, Leach A, Lievens M, Vekemans J, Dubois MC, Loucq C, Ballou WR, Cohen J, Alonso PL: Safety, immunogenicity and duration of protection of the RTS,S/AS02(D) malaria vaccine: one year follow-up of a randomized controlled phase I/Ilb trial. PLOS One 2010, 5 e13838.

2. Hoffman SL, Billingsley PF, James E, Richman A, Loyevsky M, Li T, Chakravarty S, Gunasekera A, Chattopadhyay R, Li M, Stafford R, Ahumada A, Epstein JE, Sedegah M, Reyes S, Richie TL, Lyke KE, Edelman R, Laurens MB, Plowe CV, Sim BK: Development of a metabolically active, non-replicating sporozoite vaccine to prevent Plasmodium falciparum malaria. Hum Vaccin 2010, 6:97-106.

3. Vaughan AM, Wang R, Kappe SH: Genetically engineered, attenuated whole-cell vaccine approaches for malaria. Hum Vaccin 2010, 6:107-113.

4. Kester KE, McKinney DA, Tornieporth N, Ockenhouse CF, Heppner DG, Hall T, Krzych U, Delchambre M, Voss G, Dowler MG, Palensky J, Wittes J, Cohen J, Ballou WR: Efficacy of recombinant circumsporozoite protein vaccine regimens against experimental Plasmodium falciparum malaria. $J$ Infect Dis 2001, 183:640-647.

5. Hoffman SL, Goh LM, Luke TC, Schneider I, Le TP, Doolan DL, Sacci J, de la Vega P, Dowler M, Paul C, Gordon DM, Stoute JA, Church LW, Sedegah M, Heppner DG, Ballou WR, Richie TL: Protection of humans against malaria by immunization with radiation-attenuated Plasmodium falciparum sporozoites. J Infect Dis 2002, 185:1155-1164.

6. Nussenzweig RS, Vanderberg J, Most H, Orton C: Protective immunity produced by the injection of $\mathrm{x}$-irradiated sporozoites of Plasmodium berghei. Nature 1967, 216:160-162.

7. Druilhe $P$, Rénia L, Fidock DA: Immunity to liver stages. In Malaria: parasite biology, pathogenesis, and protection.. I edition. Edited by: Sherman I. Washington DC: ASM Press; 1998:513-543.

8. Aidoo M, Udhayakumar V: Field studies of cytotoxic T lymphocytes in malaria infections: implications for malaria vaccine development. Parasitol Today 2000, 16:50-56.

9. Hay SI, Rogers DJ, Toomer JF, Snow RW: Annual Plasmodium falciparum entomological inoculation rates (EIR) across Africa: literature survey, Internet access and review. Trans R Soc Trop Med Hyg 2000, 94:113-127.

10. Wong KA, Zhou A, Rodriguez A: Protective immunity induced by daily bites from irradiated mosquitoes infected with Plasmodium yoelii. Parasite Immunol 2008, 30:482-486.

11. Casares S, Brumeanu TD, Richie TL: The RTS,S malaria vaccine. Vaccine 2010, 28:4880-4894.

12. Ocana-Morgner C, Mota MM, Rodriguez A: Malaria blood stage suppression of liver stage immunity by dendritic cells. J Exp Med 2003, 197:143-151

13. Vanderberg J: The transmission by mosquitoes of Plasmodia in the laboratory. In Malaria: Pathology, vector studies and culture. Edited by: Kreier J. New York: Academic Press; 1980:154-218.

14. Tarun AS, Baer K, Dumpit RF, Gray S, Lejarcegui N, Frevert U, Kappe SH: Quantitative isolation and in vivo imaging of malaria parasite liver stages. Int J Parasitol 2006, 36:1283-1293.

15. Smith $T A$, Leuenberger $R$, Lengeler C: Child mortality and malaria transmission intensity in Africa. Trends Parasitol 2001, 17:145-149.

16. Bruna-Romero O, Hafalla JC, Gonzalez-Aseguinolaza G, Sano G, Tsuji M, Zavala F: Detection of malaria liver stages in mice infected through the bite of a single Anopheles mosquito using a highly sensitive real-time PCR. Int J Parasitol 2001, 31:1499-1502. 
17. Kumar KA, Sano G, Boscardin S, Nussenzweig RS, Nussenzweig MC, Zavala F, Nussenzweig V: The circumsporozoite protein is an immunodominant protective antigen in irradiated sporozoites. Nature 2006, 444:937-940.

doi:10.1186/1475-2875-10-97

Cite this article as: Pollock et al:: Daily Plasmodium yoelii infective mosquito bites do not generate protection or suppress previous immunity against the liver stage. Malaria Journal 2011 10:97.

Submit your next manuscript to BioMed Central and take full advantage of:

- Convenient online submission

- Thorough peer review

- No space constraints or color figure charges

- Immediate publication on acceptance

- Inclusion in PubMed, CAS, Scopus and Google Scholar

- Research which is freely available for redistribution

Submit your manuscript at www.biomedcentral.com/submit
() Biomed Central 